\title{
Multi-Objective Optimization of Time-Cost-Quality Using Hungarian Algorithm
}

\author{
Ventepaka Yadaiah $^{1}$, V. V. Haragopal ${ }^{2}$ \\ ${ }^{1}$ Department of Mathematics, Osmania University, Hyderabad, India \\ ${ }^{2}$ Department of Statistics, Osmania University, Hyderabad, India \\ Email: v.yadaiah@gmail.com, haragopalvajjha@gmail.com
}

Received 6 December 2015; accepted 15 January 2016; published 20 January 2016

Copyright (C) 2016 by authors and Scientific Research Publishing Inc.

This work is licensed under the Creative Commons Attribution International License (CC BY).

http://creativecommons.org/licenses/by/4.0/

(c) () Op Open Access

\begin{abstract}
In this paper, we propose an algorithm for solving multi-objective assignment problem (MOAP) through Hungarian Algorithm, and this approach emphasizes on optimal solution of each objective function by minimizing the resource. To illustrate the algorithm a numerical example (Sec. 4; Table 1) is presented.
\end{abstract}

Keywords

Assignment Problem, Hungarian Algorithm, Multi-Objectives

\section{Introduction}

General assignment problem includes " $\mathrm{N}$ ” tasks that must assign to " $\mathrm{N}$ ” workers where each worker has the competence to do all tasks. However, due to personal ability or other reasons, each worker may spend different amount of resource to finish different tasks. The objective is to assign each task to a proper worker so that the total resource that spends finishing all tasks can be minimized.

Many studies have been developed to solve the assignment problem [1]-[4], like time, cost, quality and risk in construction and development projects and investment has been taken into consideration and explanation [5], projects were completed by using the FDOT to establish a model to demonstrate the functional relationship between construction cost and time for the collected highway construction projects [6]. A Multi-Objective Ant Colony Optimization is developed to analyze the advanced time cost-quality trade-off problem [7], relationship between time, cost and quality management and the attainment of client objectives [8].

Most of the developed methods for the assignment problem consider only one-objective situation, such as (1) the minimum cost assignment problem, (2) the minimum finishing time assignment problem. The minimum cost 
Table 1. Assigned cost mtrix (ACM). here cost unit: thousands, time unit: weeks, quality levels: 1, 3, 5, 7, 9.

\begin{tabular}{|c|c|c|c|c|c|}
\hline Jobs/Machine & $M_{1}$ & $M_{2}$ & $M_{3}$ & $M_{4}$ & \\
\hline \multirow[t]{3}{*}{$J_{1}$} & 9 & 7 & 4 & 6 & $\leftarrow C_{i j}$ \\
\hline & 2 & 1 & 8 & 2 & $\leftarrow T_{i j}$ \\
\hline & 1 & 1 & 1 & 5 & $\leftarrow Q_{i j}$ \\
\hline \multirow[t]{3}{*}{$J_{2}$} & 12 & 5 & 5 & 8 & $C_{i j}$ \\
\hline & 9 & 9 & 1 & 8 & $T_{i j}$ \\
\hline & 7 & 5 & 5 & 9 & $Q_{i j}$ \\
\hline \multirow[t]{3}{*}{$J_{3}$} & 9 & 9 & 9 & 11 & $C_{i j}$ \\
\hline & 8 & 9 & 5 & 6 & $T_{i j}$ \\
\hline & 1 & 7 & 5 & 7 & $Q_{i j}$ \\
\hline \multirow[t]{3}{*}{$J_{4}$} & 2 & 7 & 11 & 8 & $C_{i j}$ \\
\hline & 1 & 5 & 4 & 9 & $T_{i j}$ \\
\hline & 1 & 3 & 5 & 3 & $Q_{i j}$ \\
\hline
\end{tabular}

assignment problem focuses on how to assign tasks to workers so that the total operation cost can be minimized. Such problems have been generally discussed and well developed in many operations researches. Geetha and Nair [9] provide a solution for an assignment problem that minimizes both time and cost. Tsai et al. [10] try to solve a multi-objective decision making problem associated with cost, time, and quality by fuzzy concept. Unfortunately, the provided approaches only deal with the 2-objective assignment problem.

\section{Model Construction of Simple Assignment Problem}

Assignment problem is one of the special cases of transportation problems. The goal of the assignment problem is to minimize the cost or time of completing a number of jobs by a number of persons. An important characteristic of the assignment problem is the number of sources is equal to the number of destinations. It is explained in the following way.

1) Only one job is assigned to person.

2) Each person is assigned with exactly one job.

Management has faced with problems whose structures are identical with assignment problems.

For example, a manager has five persons for five separate jobs and the cost of assigning each job to each person is given. His goal is to assign one and only job to each person in such a way that the total cost of assignment is minimized.

Balanced assignment problem: The number of persons is equal to the number of jobs.

Minimize (Maximize):

$$
Z=\sum_{i=1}^{m} \sum_{i=1}^{n} C_{i j} X_{i j}
$$

Subject to

$$
\begin{aligned}
& \sum_{j=1}^{n} X_{i j}=1 \text {; for } i=1,2,3, \cdots, m \\
& \sum_{i=1}^{m} X_{i j}=1 \text {; for } j=1,2,3, \cdots, n
\end{aligned}
$$

where

$$
X_{i j}=\left\{\begin{array}{l}
1, \text { if the } i^{\text {th }} \text { job is assigned to the } j^{\text {th }} \text { machine. } \\
0, \text { if the } i^{\text {th }} \text { job is not assigned to the } j^{\text {th }} \text { machine. }
\end{array}\right.
$$


Problem definition: Consider a problem which consists a set of " $n$ " machines $M=\left\{M_{1}, M_{2}, M_{3}, \cdots, M_{n}\right\}$. A set of " $m$ " jobs $J=\left\{J_{1}, J_{2}, J_{3}, \cdots, J_{m}\right\}$ which is to be considered to assign for execution on " $n$ " available machines, with an execution of cost $C_{i j}($,$) , time T_{i j}($,$) , qality Q_{i j}($,$) , where i=1,2, \cdots, m$ and $j=1,2, \cdots, n$ are mentioned in the ACM of order $m \times n$, where $m=n$.

\section{Scope Triangle}

The triangle illustrates the relationship between three primary forces in an assignment. Time is available to deliver the assignment, cost represents the amount of money and quality represents fit for the purpose of assignment which should be a successful achievement.

\subsection{Methodology}

To determine the assignment of cost $(C)$, time $(T)$ and quality $(Q)$ vs. machine (s) of an assignment problem for a set of " $n$ " machines $M=\left\{M_{1}, M_{2}, M_{3}, \cdots, M_{n}\right\}$, which are to be considered as assigned for execution on " $n$ " available machines with an execution cost $C_{i j}, T_{i j}, Q_{i j}$, where $i=1,2, \cdots, m$ and $j=1,2, \cdots, n$ are mentioned in the ACM of order, where $m=n$. First of all, we obtain the sum of cost, time, and quality in each job of the ACM (Figure 1). In this way we get single objective balanced assignment problem nature (Table 2). Now we apply the Hungarian algorithm approach [11]-[13] to obtain the exact optimum solution of balanced assignment problems. To solve this problem we follow the below algorithm.

\subsection{Algorithm}

Step 1: Consider " $m$ " jobs on " $n$ " machines costs given as a matrix (ACM), which is an balanced assignment problem, where $m=n$.

Step 2: Obtain the sum of cost, time, quality in each job of the ACM.

Step 3: If the total effectiveness of ACM is to be maximized, change the sign of each cost element in the effectiveness matrix and go to Step 4, otherwise go directly to Step 5 if ACM has the total value as minimum.

Step 4: If the minimum element in the $i^{\text {th }}$ row is not zero, then subtract this minimum element from each element in the row $i(i=1,2,3, \cdots, m)$.

Step 5: If the minimum element in the column $\mathrm{j}$ is not zero, then subtract this minimum element from each element in the column $j(j=1,2,3, \cdots, m)$.

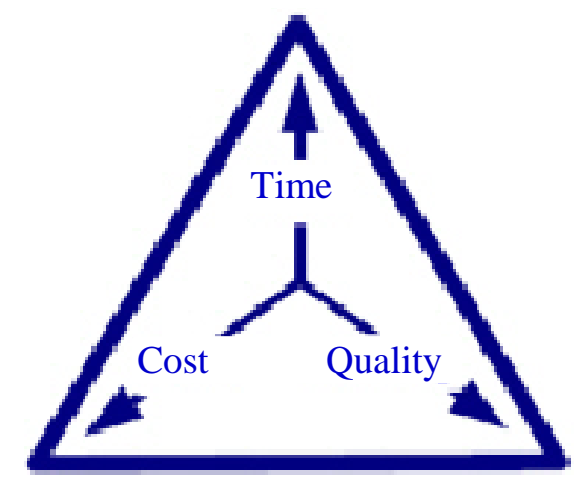

Figure 1. Scope-Triangle.

Table 2. Modified ACM (,).

\begin{tabular}{ccccc}
\hline & $M_{1}$ & $M_{2}$ & $M_{3}$ & $M_{4}$ \\
\hline$J_{1}$ & 12 & 9 & 13 & 13 \\
$J_{2}$ & 28 & 19 & 11 & 25 \\
$J_{3}$ & 18 & 25 & 19 & 24 \\
$J_{4}$ & 4 & 15 & 20 & 20 \\
\hline
\end{tabular}


Step 6: Examine rows successively, beginning with row 1, for a row with exactly one unmarked zero. If at least one exists, mark this zero with the symbol $(\square)$ to denote an assignment. Cross out $(\mathrm{X})$ the other zeros in the same column so that additional assignment will not be made to that column. Repeat the process until each row has no unmarked zeros or at least two unmarked zeros.

Step 7: Examine columns successively, beginning with column 1, for a column with exactly one unmarked zero. If at least one exists, mark this zero with the symbol ( $\square$ ) to denote an assignment. Cross out (X) the other zeros in the same row so additional assignment will not be made to that row. Repeat the process until each column has no unmarked zeros or at least two unmarked zeros.

Step 8: Repeat Steps 7 and 8 successively (if necessary) until one of the three things occurs:

Step 9: Every row has an assignment ( $\square$ ). Go to Step 16.

Step 10: There are at least two unmarked zeros in each row and each column. Go to Step 7.

Step 11: There are no zeros left unmarked and a complete assignment has not been made. Go to Step 10.

Step 12: Check $(\sqrt{ })$ all rows for which assignment $(\square)$ has not been made.

Step 13: Check $(\sqrt{ })$ columns not already checked which have a zero in checked rows.

Step 14: Check $(\sqrt{ })$ rows not already checked which have assignments in the checked column.

Step 15: Repeat Steps 11 and 12 until the chain of checking ends.

Step 16: Draw lines through all unchecked rows and through all checked columns. This will necessarily give the minimum number of lines needed to cover each zero at least one time.

Step 17: Examine the elements that do not have at least one line through them. Select the smallest of these and subtract it from every element in each row that contains at least one uncovered element. Add the same element to every element in each column that has a vertical line through it. Return to Step 7.

Step 18: List the assignment cost and combination corresponding to sub problem.

Step 19: Add assignment cost of each sub problem to obtain the total assignment cost of the main problem, which shall be the optimal cost, and also rearrange the combinations.

Step 20: Stop.

\section{Illustration}

Solve the problem (Table 1) assuming that the objective is to minimize the total cost, time and quality.

Now obtain the sum of cost, time, quality in each job of the ACM and we get modified ACM (,) (Table 2).

Now apply the Hungarian method for Modified ACM (,), then the final optimal assignments of Modified ACM (,) (Table 2) as follows:

$$
J_{4} \rightarrow M_{1}, \quad J_{1} \rightarrow M_{2}, \quad J_{2} \rightarrow M_{3}, \quad J_{3} \rightarrow M_{4}
$$

\section{Conclusion}

The above illustration was taken by the defined algorithm and implemented on several sizes of the problems to test the effectiveness of the algorithm. This approach was implemented on different sizes of multi-objective balanced assignment problems from the above. We noticed that by using standard Hungarian method we could get the optimum value. The time complexity verified and found that they were getting optimum in less time comparative to other methods.

\section{References}

[1] Winston. L.W. (1991) Operations Research: Applications and Algorithm. Pws-Kent Puublishing Company, Boston.

[2] Hillier. S.F. and Lieberman, J.G. (2001) Introduction to Operation Research. McGraw-Hill, Boston.

[3] Taha, H.A. (1971) Operation Research: An Introduction. MacMillan Inc., New York.

[4] Ravindran, A. and Ramaswami, V. (1977). On the Bottleneck Assignment Problem. Journal of Optimization Theory and Applications, 21, 451-458. http://dx.doi.org/10.1007/BF00933089

[5] Rezaian, A. (2011) Time-Cost-Quality-Risk of Construction and Development Projects or Investment. Middle-East Journal of Scientific Research, 10, 218-223.

[6] Shr, J.-F. and Chen, W.-T. (2006) Functional Model of Cost and Time for Highway Construction Projects. Journal of Marine Science and Technology, 14, 127-138. 
[7] Afshar, A., Kaveh, A. and Shoghli, O.R. (2007) Multiobjective Optimization of Time-Cost-Quality Using Multi-Colony ant Algorithm. Asian Journal of civil Engineering (Building and Housing), 8, 113-124.

[8] Bowen, P.A., Hall, K.A., Edwards, P.J., Pearl, R.G. and Cattell, K.S. (2002) Perceptions of Time, Cost, Quality Management on Building Projects. The Australian Journal of Construction Economics and Building, 2, 48-56.

[9] Geetha, S. and Nair, K.P.K. (1993) A Variation of the Assignment Problems. European Journal of Operational Research, 68, 422-426. http://dx.doi.org/10.1016/0377-2217(93)90198-V

[10] Tsai, C., Wei, C.-C. and Cheng, C.-L. (1999) Multiobjective Fuzzy Deployment of Manpower. International Journal of the Computer, the Internet and Management, 7,.

[11] Pandit, S.N.N. (1963) Some Quantitative Combinatorial Search Problems. PhD Thesis, IIT Khargpur.

[12] Ramesh, M. (1997) Lexi-Search Approach to Some Combinatorial Programming Problem. PhD Thesis, University of Hyderabad, Hyderabad.

[13] Gillett Billy, E. (2000) Introduction to Operations Research-A Computer Oriented Algorithm Approach. Tata McGraw Hill, New Delhi. 